\title{
CAMBIOS EN LOS HÁBITOS DE COMPRA EN PANDEMIA DE LA CANASTA DE CUIDADO PERSONAL EN LOS HOGARES
}

\section{CHANGES IN PANDEMIC SHOPPING HABITS OF THE HOME CARE BASKET}

\author{
Silvia Astudillo Baldeón ${ }^{1}$, Karla Bolaños Flor $^{2}$
}

\section{Palabras clave: Comportamiento del consumidor, hábitos de consumo}

\begin{abstract}
Resumen
El presente artículo permite conocer los cambios en los hábitos de compra en pandemia de la canasta de cuidado personal en los hogares basado en las decisiones de compras de los usuarios que es un proceso que cambia por factores internos o externos. Para un correcto análisis se usó información cuantitativa proporcionada por Kantar división Worldpanel Ecuador que hace investigaciones de mercado periódicas a 1.706 hogares a nivel nacional para determinar sus comportamientos de compra en diversas categorías. La información cualitativa se obtuvo de la realización de tres grupos focales que tuvieran características similares a los hogares auditados por Kantar para conocer los motivos de los cambios en sus hábitos de compra durante la pandemia. Los principales hallazgos obtenidos son que la vanidad paso a segundo plano, la salud y la alimentación son la prioridad además de que los gastos no han disminuido, sino que han sido redistribuidos.
\end{abstract}

Códigos JEL: D1 1

\footnotetext{
${ }^{1}$ ESAI Business School, Universidad Espíritu Santo (Ecuador). E-mail: sastudillo@uees.edu.ec

${ }^{2}$ ESAI Business School, Universidad Espíritu Santo (Ecuador). E-mail: karlabolanos@uees.edu.ec
} 


\section{INTRODUCCIÓN}

Ante la noticia mundial en febrero de 2020 por parte de la OMS, el mundo atraviesa nuevamente una pandemia después de 100 años, afectando a nivel global tanto en lo económico como en lo social. Este estudio busca entender el por qué cambió el comportamiento de compra del hogar en las categorías de cuidado personal.

Con la llegada del Covid-19, muchas industrias están enfrentando una crisis económica. Se había pronosticado que iba a ser un año de grandes ventas y expansión, pero la pandemia sigue cobrando vidas y la economía global continúa en declive. Una de las industrias que más se ha visto afectada es la cosmética, ya que en la pandemia los consumidores empezaron a cambiar sus intereses y hábitos por completo, priorizando productos de cuidado personal, alimentos y productos de aseo (Suprapak, 2020).

Los ecuatorianos como el resto del mundo, atravesó esta noticia con temores ante el desconocimiento y desconcierto al confinamiento, sin fecha de término este encierro, recomendado por la OMS (2020). En Sudamérica las medidas tomadas tuvieron afectaciones tanto económicas como sociales, generando con ellos ajustes en el comportamiento de los consumidores; donde la priorización al gasto de categorías prioritarias o vinculadas a salud y primera necesidad crecieron al ritmo de doble dígito, pero en cambio por otro lado hay categorías que no fueron considerados en la recompra en periodos de confinamiento.

Lo expuesto se confirma en el artículo publicado por el Fondo Monetario Internacional (2020) donde se evidencia que en Ecuador hubo una caída de los ingresos y un aumento de los gastos en los hogares producto de la pandemia donde la prioridad era la salud.

Es fundamental comprender a los consumidores para conocer el origen de sus cambios de hábitos de consumo por eso es importante indagar en los mecanismos de compra, el motivo de compra, que es esencial comprar, si existen preferencias por precios, calidad, marca $u$ otro factor de compra al momento de elegir uno o varios productos de cuidado personal antes, durante y después de la pandemia por temas de tendencia.

Por lo expuesto con anterioridad el objetivo de este artículo es determinar los cambios en los hábitos de compra en pandemia de la canasta de cuidado personal en los hogares por medio del análisis de información cuantitativa y cualitativa

Es importante conocer el comportamiento de compra del consumidor a nivel general y de la categoría de cuidado personal para identificar que tan sensible son las mismas antes situaciones externas incontrolables como una pandemia y tener idea de que estrategias de marketing implementar para mantener el comercio.

\section{REVISIÓN DE LITERATURA}

La revisión literaria se divide en tres macro conceptos que son decisión de compra, proceso de compra e indicadores, mecanismo de venta y estrategias de Marketing.

\section{Decisión de compra}

Este proceso nace de las necesidades que tienen las personas que de acuerdo con la teoría de Maslow se dividen en cinco categorías estas surgen de manera innata y busca satisfacer las necesidades por orden jerárquico o prioritario (Espinosa, 2019). Comprendiendo las necesidades se puede entender el comportamiento del consumidor que se basa en las actitudes que se ven influenciados por factores internos y externos que perciben de un producto o servicio enfocados en la empresa, la reputación, la percepción y los anuncios que influyen en su manera de comprar (Sordo, 2020).

Del comportamiento se derivan los hábitos de consumo que son las tendencias que tienen los consumidores al comprar productos y servicios que se ven influenciados por diversos factores que son internos y externos, los primeros se basan en sus deseos, necesidades y preferencias de compra se relacionan con sexo, edad, ubicación geográfica, nivel educativo, familia, estado de ánimo, mientras que los externos son aspectos que influyen en la compra pero no están relacionados 
a las necesidades o deseos individuales sino que surgen de anuncios publicitarios, de recomendaciones de famosos, de testimonios de otros usuarios y de tendencias actuales (QuestionPro, 2021).

Es importante mencionar que cuando no existe una necesidad clara las empresas deben crear la necesidad por medio de diversos mecanismos publicitarios para asegurar las ventas de sus productos o servicios para esto se pueden basar en las actividades antes, durante y después de la compra.

De acuerdo a Solomon (2013) existen los siguientes aspectos relacionados con las actividades que se realizan antes, durante $y$ después de la compra, en la etapa antes de la compra intervienen factores situacionales. Contextos de uso, presión de tiempo, estado de ánimo y la orientación hacia la compra; durante la compra intervienes aspectos como la experiencia de compra, estímulos en el punto de venta y la interacción durante la venta y finalmente después de la compra los factores que intervienen son la satisfacción del consumidor, el desecho del producto y los mercados alternativos.

\section{Proceso de decisión de compra}

Es la evolución por las distintas fases por las que pasa el consumidor y que van desde que reconoce que tiene una necesidad hasta que finalmente decide comprar (Rodríguez, 2019). Para comprender el proceso de decisión de compra es necesario conocer las etapas que tienen los consumidores durante el mismo según la EAE Business School (2021) esta se basa en reconocer la necesidad esta etapa nace en los consumidores por una influencia interna o externa que los motiva a buscar una solución para una necesidad, la segunda es buscar información se basa en investigar por medio de distintas fuentes si existe un producto o servicio que satisfaga su necesidad, se arma una lista de posibles vendedores.

La tercera etapa es evaluar distintas opciones en esta fase se compara la información obtenida y se evalúa cada vendedor, muchos consumidores revisan opiniones de expertos o de clientes para poder confirmar que la empresa investigada ofrece un producto o servicio de calidad, si la inversión a realizar es alta tomará más tiempo buscar la información y comparar la misma, la cuarta es la decisión de compra esta etapa se da luego de comparar toda la información obtenida y se decide comprar un determinado bien o servicio. La última etapa es evaluar el producto o servicio es la etapa final donde el consumidor evalúa o comprueba que el producto o servicio adquirido cumple con sus expectativas y satisface sus necesidades.

Todas las decisiones de compra están relacionadas a factores que pueden influir en el mismo según Sordo (2020) los factores internos que influyen se basan en las características del cliente potencial a captar son parte de su personalidad, son fácil de reconocerlos a primera vista, se basan en sus hábitos como consumidor sean estos locales es decir se relacionan a su país o idioma, personales se basa en su experiencia y el estilo de vida que son las decisiones y preferencias que lo definen en el día a día.

Los factores externos que influyen en la decisión de compra son ajenos al consumidor como aspectos administrativos que se basan en leyes que influyen en la decisión de compra, públicos basados en recomendaciones por conocidos y eventos imprevistos que son situaciones que cambian la toma de decisiones.

Es fundamental mencionar que las decisiones de compra se clasifican por tipos de comportamientos según QuestionPro (2021) existen cuatro comportamientos de compra que son compra compleja cuando se adquiere un producto caro que no conoce y consulta a otras personas, compra que reduce la disonancia cuando se compra un producto, pero se tiene miedo de haber hecho una mala elección, compra habitual cuando se compra un producto como parte de su vida diaria y compra por búsqueda de variedad cuando se busca probar nuevos productos.

\section{Indicadores, mecanismo de venta y estrategias de marketing}

La finalidad de toda empresa es conocer el comportamiento del cliente y el proceso de 
compra que realizan los mismos para poder establecer estrategias que le permitan vender sus productos o servicios enfocados en satisfacer una necesidad para una correcta evaluación de sus niveles de ventas presupuestadas se utilizan indicadores de medición de compras en marketing son aquellos indicadores que definimos como necesarios para valorar la efectividad de la acción (Viñarás, 2021).

Uno de los indicadores más utilizados es la frecuencia de compra que es la cantidad de veces que un cliente promedio compra un producto a una sola marca en un período determinado (Camargo, 2020). Otro indicador es el ticket promedio de compra que es el valor promedio o el promedio de compras que cada cliente hace en un negocio o producto, por medio de un establecimiento físico o por medio online (Nonato, 2019). Otras empresas utilizan la penetración de compra que es un indicador que relaciona el total de clientes que nos compran del total de una población (García, 2018).

Todos los indicadores mencionados los utilizan las empresas con la finalidad de satisfacer las necesidades de los clientes que permiten identificar las debilidades de la empresa y hacer los cambios necesarios para retener al público existente y atraer nuevos clientes (Centro Europeo de Postgrado, 2021).

Es fundamental conocer cuáles son los canales de ventas en los que pueden ingresar las diversas empresas para poder ofrecer sus productos y servicios, teniendo canales físicos y electrónicos (Hotmart, 2021). Los canales físicos se basan en puntos de ventas como tiendas especializadas, supermercados, tiendas de barrios entre otros puntos que permiten tener al cliente una experiencia más amplia por el contacto directo con los asesores de ventas (Cera, 2021).

Dentro de los canales físicos se encuentran los tradicionales que están representados por las tiendas de barrio, es el comercio más familiar, son puntos de venta que comercializan alimentos, bebidas y artículos de higiene y limpieza (Sima, 2020).
Otro canal físico es el supermercado que es un lugar donde se compran y venden productos de alimentación, higiene y cuidado personal, entre otros productos, estos lugares tienen amplia cobertura (Peiró, 2020)

Y otro canal físico muy utilizado es el catálogo que es un modelo de negocio para vender productos o servicios, en donde el comprador ve y compra los productos a través de un catálogo. En este sistema el vendedor realiza sus ventas visitando personalmente al cliente potencial o utilizando otro tipo de herramientas de marketing directo, tales como el teléfono, Internet, correo regular y electrónico u otros medios (Bellash, 2015).

Los Canales electrónicos son el modelo de negocios basados en las transacciones de productos y servicios en los medios electrónicos, ya sean en las redes sociales o en los sitios web (Higuerey, 2019). Dentro de los canales electrónicos se destacan los siguientes que son los que tienen mayor afluencia.

Marketplace que es una vitrina o tienda virtual en la que los clientes pueden tener acceso a productos de diversas marcas o empresas (Hotmart, 2021), las páginas o sitios web que son medios de comunicación por medio de navegadores portátiles que permiten transmitir mensajes y crear comunidad por el uso de audio, vídeo, texto y sus diferentes combinaciones, que tienen como finalidad ser fuentes de información o mecanismos de ventas (Peiró, 2019) y las redes sociales que tienen como finalidad crear vínculos sea por temas comerciales, amistad, trabajo, parentesco, entre otros (Rockcontent, 2019).

Con la nueva normalidad para poder captar y mantener a los clientes es fundamental para las empresas establecer estrategias de publicidad que tienen objetivos es posicionar una marca a través de diferentes canales (Sordo, 2021). Para esto se aplican las siguientes estrategias publicitarias:

Marketing de contenidos es una manera de involucrar a tu público objetivo y hacer crecer tu red de leads y clientes a través de la creación de contenidos relevantes y valiosos, atrayendo, involucrando y generando valor para las personas 
$y$, de esa forma, crear una percepción positiva de tu marca, generando más negocios (Giraldo, 2019).

Marketing de redes sociales se basa en posesionar un negocio a través de videos, fotos y blogs con el fin de dar exposición a una empresa y ejecutar distintos procesos de compras (Peralta, 2021) esta estrategia se la complementa con concursos y sorteos que permiten aumentar el número de seguidores y el nivel de interacción. (Cardona, 2019),

La publicidad pagada por medio de redes sociales con diversos fines como generar visitas, interacciones, seguidores $\mathrm{o}$ dar a conocer promociones es un mecanismo muy utilizado (Campaña, 2021), esta estrategia se complementa con el marketing de influencers que consiste en lograr una serie de vínculos de colaboración entre marcas o empresas y aquellas personas con una gran visibilidad y protagonismo en internet, a las que se conoce como 'influencers' (InboundCycle, 2021).

Las estrategias antes detalladas son en canales electrónicos, pero se siguen manteniendo estrategias tradicionales como promociones que se basa en crear estímulos que pretenda provocar una respuesta determinada en un público objetivo a corto plazo, ejemplo de estos mecanismos son los precios de lanzamiento, obsequios, muestras gratis, $2 \times 1,3 \times 2$, descuentos en el precio, cupones entre otras estrategias promocionales. (Nardi, 2017)

Los descuentos es una acción por medio de la cual se reduce el precio de un determinado producto en un cierto porcentaje para captar una venta por volumen (Ramírez, 2018) y las pruebas gratis también es una estrategia ideal para el lanzamiento de nuevos productos, especialmente si se trata de artículos de cosmética o de higiene personal (Emprendedores.es, 2021). La estrategia más importante son las recomendaciones que busca aprovechar la experiencia de los clientes y motivarlos a contar su experiencia a amigos, familiares o colegas de trabajo. La gente confía en quienes son cercanos y por ello también se fía de la recomendación de un producto o la contratación de un servicio si proviene de una fuente cercana y directa. Los reviews de expertos en internet también permiten adquirir los productos. (Pursell, 2020).

La pandemia obligó a todos a digitalizarse tantos clientes como empresas por lo cual es fundamental tener presencia en redes sociales y aplicar estrategias adecuadas de marketing que permitan mantener a los clientes actuales y captar nuevos.

De acuerdo con el reporte anual The Global State of Digital in 2019 creado por Hootsuite y We Are Social, el 52\% de la población mundial utiliza redes sociales. Esta enorme audiencia global que utiliza estos canales representa un mercado vasto de oportunidad para cualquier empresa, sin importar su tamaño. Una oportunidad para crear las estrategias de marketing que permitirán que el alcance de tu negocio se extienda al mundo digital (Hootsuite, 2019).

De acuerdo a todos los conceptos expuestos todo nace de una necesidad que se encuentra dentro de la pirámide de Maslow, que busca ser satisfecha para lo cual los individuos buscan por diversos mecanismos información de productos o servicios que logren satisfacer su necesidad, nace el proceso de compra que pasa por una serie de etapas donde intervienen una serie de factores internos y externos que permiten al cliente potencial decidir volverse consumidor y adquirir determinado bien o servicio.

Se puede concluir que las empresas por medios de sus canales de ventas físicos o de comercio electrónico deben de aplicar una serie de estrategias de marketing enfocadas a la publicidad que le permita vender sus productos, crear una comunidad para fidelizar su marca y mantener a sus clientes actuales además de conseguir nuevos clientes por la interacción de su comunidad.

\section{METODOLOGÍA}

El estudio realizado es de carácter exploratorio que corresponde al primer acercamiento a un tema específico antes de abordarlo en un trabajo investigativo más 
profundo. Se trata de un proceso para tener información básica relacionada con el problema de investigación. (Ortiz, 2020).

Para poder comprender los cambios en los hábitos de compra y sus usos en período de pandemia de los productos de la canasta de cuidado personal en los hogares ecuatorianos, se plantea analizar bajo dos metodologías la información como son: home panel para la parte cuantitativa con muestra probabilística y el focus group para obtener los resultados cualitativos.

Home panel está conformado por un grupo de encuestados que aceptan voluntariamente formar parte de una investigación y contribuir con el proceso de recolección de datos que lleva a cabo una organización (QuestionPro, 2021). Focus group es una metodología cualitativa con el objetivo de identificar el motivo de los cambios de las ventas y recompras de las canastas analizadas con mayor contracción, la herramienta es un focus group que es un método de investigación de mercado que tiene la función de analizar y captar feedbacks sobre productos, servicios y campañas de marketing de una empresa (Lisboa, 2019).

Bajo estas dos metodologías se podrá tener una sinergia de información, las cuales permitirán tener el QUÉ sucedió en el comportamiento real de compra y POR QUÉ se debió estos cambios bajo la indagación grupal.

Para comprender la información cuantitativa y cualitativa es necesario acudir a la analítica de datos que se basa en analizar, procesar, categorizar y utilizar información sobre los procesos de una empresa, tendencias, rasgos de los usuarios y otros datos que permitan comprender un tema en específico (Peña, 2020).

Para comprender mejor la analítica de datos es necesario crear gráficos que permiten de forma visual comprender mejor la información numérica obtenida de la base de datos de la empresa Kantar (Pérez \& Merino, 2021).

La información cuantitativa fue proveída por la empresa Kantar División Worldpanel, la cual proyecta el comportamiento de compra de la canasta de consumo masivo industrializada, con 70 categorías las cuales la dividen en 6 canastas para comprender la priorización de compras en los periodos de análisis. La información proyectada es obtenida bajo una muestra de 1706 hogares, los cuales están divididos en 500 hogares en Quito y 500 en Guayaquil, más los conglomerados de Resto Costa y Resto Sierra. Esta muestra fue dispersa y alineada a la estructura poblacional del INEC del año 2000.

La muestra representa el $91 \%$ de cobertura urbana y 55\% poblacional. Para obtener los resultados proyectados, Kantar tiene una recolección semanal de las compras físicas donde las amas de casas las cuales están en la confección de la muestra presentan los envases con etiquetas de las compras semanales como sustento de los registros y las compras en autoservicios deben además deben tener el recibo (desde marzo 2021 por pandemia es de forma virtual-telefónica).

El proceso de recolección una vez concluido, se ingresa todos los actos de compras y detalles requeridos y se procesan las bases de datos la cual bajo el programa exclusivo de Kantar se permite obtener toda la data para su análisis y asesoramiento.

La metodología de Home panel, permite identificar bajo sus variables el comportamiento de compra de los hogares como penetración que se identifica de forma porcentual, la frecuencia de compra al tener la periodicidad de audición, además que se registra el detalle de los productos físicos, la frecuencia de compra, las unidades compradas en el acto, la compra por acto, el ticket de la transacción y el precio promedio pagado por el gramaje de las categorías recabadas.

Se analizó el comportamiento de compra de la muestra que proyecta Kantar con sus 70 categorías en los últimos 3 años, con énfasis en 3 períodos para definir el impacto de las de canastas de mayor contracción que evidencian el ajuste del comportamiento de compra, influenciado por la pandemia.

La segunda herramienta es el Focus Groups que se lo realizó a tres grupos teniendo en cuenta que cada uno debía estar conformado por 6 u 8 
personas, con una duración de 1-2 horas, a través de las plataformas de comunicación virtual como son Google Meets o Teams, con la finalidad de obtener información relevante. La moderadora fue Karla Bolaños, quién tenía conocimientos por su trabajo para realizar esta labor.

Con la finalidad de que todos los grupos sean homogéneos es decir que tengan características similares se establecieron los siguientes filtros para poder cumplir con la premisa antes mencionada.

Los grupos se dividían por estratos económicos teniendo como base la información proporcionada por el INEC (2011) que especifica que en el país existen cinco niveles económicos que son A, B, C+, C- y D, esto se lo alineo a la metodología de Kantar agrupando a los estratos $\mathrm{A}+\mathrm{B}, \mathrm{C}$ y $\mathrm{D}+\mathrm{E}$ con la finalidad de obtener datos más significativos. Los miembros del grupo focal tenían que ser mujeres de entre 20 hasta 70 años de edad que permitiría tener una mayor comprensión de los cambios en los hábitos de consumo desde diversas perspectivas por tener varios grupos de edades. La ocupación y su estado civil también fue indistinto debido a que se buscó cubrir todos los aspectos para comprender los cambios de consumo de forma general y no especifica para evitar sesgos en la investigación realizada.

Con la finalidad de motivar a las participantes de estrato bajo y medio a participar se les otorgó un incentivo como entradas al cine o recargas de celular para poder captar su atención y que brinden información relevante para la investigación. La finalidad de los grupos focales está en contrastar la información cuantitativa obtenida de la empresa Kantar y poder identificar qué tipo de comportamiento han tenido las consumidoras de la canasta de productos de cuidado personal durante los periodos de interés bajo una guía de preguntas (Hamui-Sutton \& Varela-Ruiz, 2013).

La información obtenida de los grupos focales es analizada mediante el análisis de contenido por grupo donde se identifican comportamientos o hábitos de compra y estos se comparan con los otros grupos para identificar un patrón de consumo.

Para la guía se usó de referencia el esquema de preguntas elaborado por la empresa Kantar división Insight, el cual tenía más de 84 preguntas, sin embargo, por medio de un panel de expertos se redujo a 30 las interrogantes, la cual va desde lo general hasta lo específico.

\section{RESULTADOS}

Para poder evaluar los cambios en los hábitos de consumo fue necesario contrarrestar el dato cuantitativo proporcionado por la empresa con el dato cualitativo proporcionado por la herramienta de grupos focales por estratos económicos teniendo los siguientes datos.

\section{Análisis cuantitativo enfocado en los datos proporcionados por la empresa Kantar}

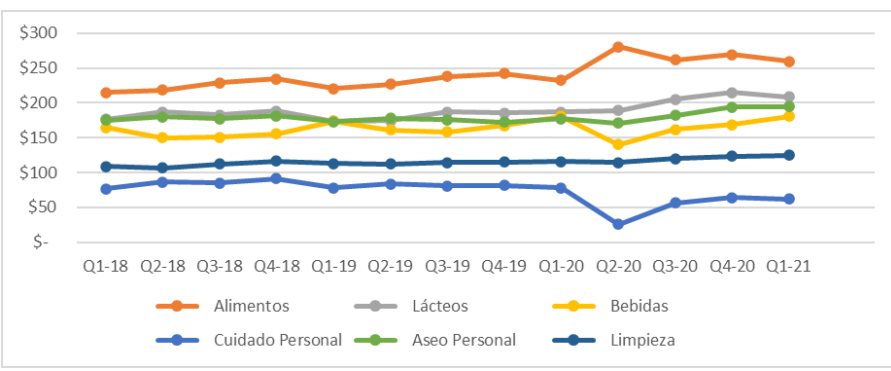

Figura 1. Tendencia trimestral en Millones de 2018 a 2021 de las canastas.

Elaborado por: Las autoras extraídas de los datos de Kantar división Worldpanel.

De acuerdo con la figura 1 se observa que la canasta que más creció fue alimentos debido a que el confinamiento hizo que los ecuatorianos se abastezcan de productos para su uso diario teniendo un pico alto en el segundo trimestre del 2020 y continua en crecimiento debido a que la alimentación tiene un papel importante para evitar contagios (Tetrapack, 2020).

El consumo de productos básicos se incrementó de manera importante, la gente compra para consumir y para guardar, al mismo tiempo busca productos más sanos y de mayor duración, porque la movilidad está reducida (El Universo, 2020). 
La canasta de lácteos y de aseo personal tuvieron un crecimiento sutil pero los productos de aseo personal no fueron tan demandados en el segundo trimestre del 2020 luego recuperaron participación por promociones publicitarias como $2 \times 1$, descuentos, productos gratis entre otras estrategias de ventas.

La canasta de bebidas tuvo una reducción considerable en sus ventas en el segundo trimestre del 2020 y ha logrado recuperar su participación de acuerdo a la información del primer trimestre del 2021 se puede asumir que esta recuperación se debe a la nueva normalidad por la apertura de restaurantes, hoteles y de los trabajos.

La canasta de limpieza ha tenido un ligero crecimiento trimestre a trimestre esto se debe a que por temas de evitar contagios se busca limpiar constantemente el hogar por recomendaciones de expertos y conocidos (BASF, 2020).

Finalmente se observa que la canasta de cuidado personal fue la más afectada en el segundo trimestre del 2020 y no ha logrado recuperar su valor de ventas hasta el primer trimestre del 2021 esto se debe a que la vanidad paso a un segundo plano, en la actualidad la salud y alimentación es lo esencial (Stanpa, 2021).

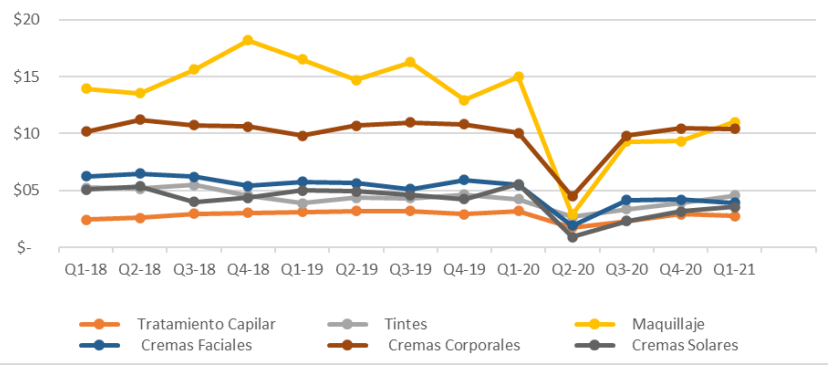

Figura 2. Tendencia trimestral en Millones de 2018 a 2021 canasta de cuidado personal Elaborado por: Las autoras extraídas de los datos de Kantar división Worldpanel.

Según la figura 2 dentro de la canasta de cuidado personal las categorías más afectadas en sus ventas son fragancias y maquillaje que no han logrado recuperar su nivel de ventas mientras que las otras categorías tuvieron un impacto en el mismo trimestre, pero han logrado recuperar sus ventas parcialmente.
La recuperación de las otras categorías se dio por una mayor preocupación por el cuidado de la piel debido a que el uso de la mascarilla reseca la piel por lo cual necesitan hidratar la misma, ahora la tendencia es ser más natural esto se debe a que la mascarilla no permite que se aprecie el maquillaje.

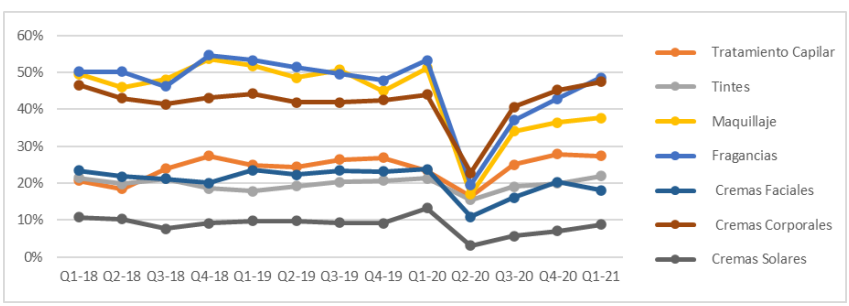

Figura 3. Penetración en porcentaje por trimestre del 2018 al 2020 en las categorías de la canasta de cuidado personal

Elaborado por: Las autoras extraídas de los datos de Kantar división Worldpanel.

En la figura 3 se observa que en el primer trimestre del 2020 casi todas las categorías tuvieron crecimiento esto se puede asociar a que es inicio de temporada playera y a festividades como Día de Reyes, San Valentín y Día de la mujer donde se dan regalos.

En el segundo trimestre donde el confinamiento es una realidad todas caen perdiendo su participación en el mercado debido a que estos productos dejaron de ser demandados por no ser prioritarios, se confirma que fragancia y maquillaje son las categorías más afectadas que no han logrado recuperarse tras la pandemia.

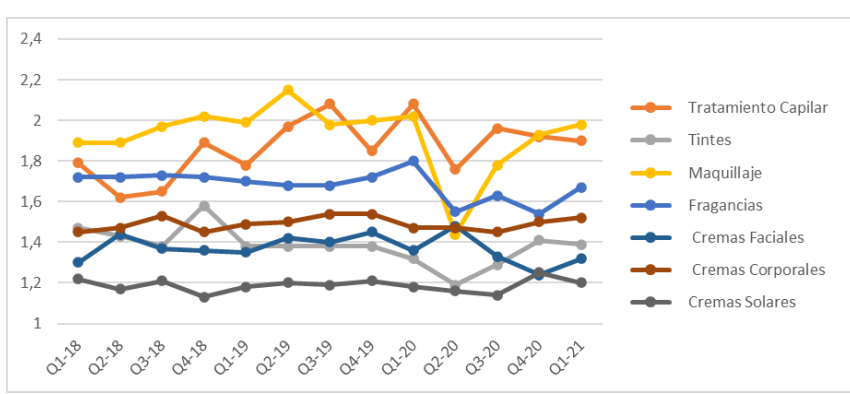

Figura 4. Frecuencia de compra por trimestre del 2018 al 2020 en las categorías de la canasta de cuidado personal.

Elaborado por: Las autoras extraídas de los datos de Kantar división Worldpanel. 
La frecuencia de compra de la categoría de maquillaje fue la más afectada se evidencia una recuperación en la frecuencia, pero esto se debe a las estrategias promocionales que han manejado diversas marcas con la finalidad de incentivar a la compra y recuperar sus ventas.

El resto de las categorías disminuyó su frecuencia en el segundo trimestre del 2020 y ha aumentado ligeramente la misma en los trimestres posteriores debido a la generación de promociones como descuentos, $2 \times 1$, productos gratis, mayor contenido entre otros aspectos que han impulsado la recompra

El canal de ventas más utilizado en esta canasta es la venta por catálogo que tenía una participación entre el $64 \%$ y $67 \%$, producto de la pandemia y de las restricciones de movilidad esta participación se redujo entre 6 a $17 \%$, esa participación la captó las ventas por medio de mecanismos electrónicos y supermercados.

\section{Análisis cualitativo enfocado por estratos socioeconómicos}

En el estrato económico bajo las entrevistas en su mayoría son amas de casas y mujeres que realizan trabajos de forma independiente con edades comprendidas de 25 a 55 años, están manifestaron lo siguiente:

El ingreso percibido por su unidad económica por la pandemia se vio afectado debido a que estos disminuyeron a causa de que dejaron de vender sus productos o servicios, o algún miembro de su familia dejo de trabajar lo que les ha cambiado sus hábitos de compras.

La frecuencia de compras de los productos para su hogar ha disminuido algunas compraban antes de forma diaria ahora compran de forma semanal, quincenal o mensual para prevenir algún contagio para salvaguardar la salud de su familia.

La pandemia a cambiado la rutina de todos ahora es primordial la seguridad de la familia por lo cual se compran más productos de higiene personal y del hogar como desinfectantes, alcohol, gel, cloro, jabón entre otros que permiten tener un mayor cuidado siguiendo las recomendaciones de entidades públicas que es el lavado frecuente de manos y el limpiar sus hogares para evitar que el virus pueda estar en el ambiente.

Todas las personas manifiestan que han reducidos sus gastos destinados a ocio, entrenamiento y alimentación fuera de sus hogares debido a que prefieren ahorrar dinero para una emergencia en caso de que algún familiar se enferme debido a que conocen que el tratamiento de la enfermedad causada por el covid-19 es alto.

Creen que sus gastos se han mantenido o han crecido levemente debido a que han redireccionado el gasto de ciertos productos como ropa, maquillaje, perfumes, la compra de ciertos dulces o comida que pueden realizarlos en casa, priorizaron la adquisición de alimentos básicos y productos de aseo, medicamentos y gastos en educación.

Todas dejaron de comprar maquillaje y en menor proporción fragancias debido a que no salen y las mascarillas les tapa parte de su rostro además de que si se maquillan todo el producto se sale por el uso de la mascarilla, lo consideran un desperdicio de dinero.

Las entrevistadas usan más cremas faciales sean estas de día, de noche o de uso diario, protectores solares, debido a que es importante evitar la resequedad y cuidar su piel por el uso de la mascarilla o por el estar mucho tiempo en casa sin exposición al ambiente.

Otro hallazgo significativo es que aumentaron el gasto en pasta dental, jabón de tocador, shampoo y mantuvieron el gasto en papel higiénico y desodorantes, el aumento de los productos antes mencionados es por temas de cuidado y por prevenir la infección por el virus en la actualidad se bañan más veces y se lavan las manos con mayor frecuencia.

La rutina de maquillaje cambio significativamente en la actualidad ya no se maquillan tanto debido a que no pueden salir ahora prefieren el maquillaje natural resaltan que cuando salen ahora buscan resaltar su mirada por 
medio de rímel, pestañas o el maquillarse sus cejas.

Para poder adquirir productos de belleza se fijan en promociones, precio, calidad, funcionalidad y el tema de que los productos sean libres de crueldad animal, esos son los factores diferenciadores para adquirir sus productos, algunas aseguran que se mantienen comprando las mismas marcas, otras cambiaron de marcas debido a que ahora prefirieron comprar productos de calidad y otras no ven marcas sino el tema de beneficios.

Los canales de compra que utilizan actualmente son por medio de mecanismos digitales donde diversos emprendimientos proporcionan el catálogo de los productos que venden por medios de enlaces que les permite ver temas de precios y beneficios, y cuando tienen recursos compran en tiendas especializadas los productos de belleza y maquillaje.

Las entrevistadas del estrato económico bajo manifestaron que cuando se termine la pandemia creen que volverán a utilizar maquillajes es decir volverán a su rutina de maquillaje antes de pandemia usarán más bases líquidas y en polvo, labiales, rubores y otros productos que dejaron de usar debido a que la mascarilla no se los permite.

En el estrato económico medio se entrevistaron a cuatro mujeres que se encuentran trabajando y tres mujeres que ahora no tienen trabajo y son amas de casa con edades comprendidas de 27 a 68 años, están manifestaron lo siguiente:

El ingreso percibido en muchos casos se mantuvo debido a que continúan trabajando mientras que para las personas que se quedaron sin trabajo tenían sus ahorros y el dinero de su liquidación o el soporte de algún miembro de su familia que mantiene el hogar.

La pandemia les permitió acercarse más a sus familiares y a Dios debido a que comprendieron que lo único importante es la salud y que sin ella no se puede lograr cumplir ciertas aspiraciones personales.
La frecuencia de compra cambio ahora prefieren adquirir los productos necesarios para su diario vivir de manera mensualizada en su mayoría debido a que para resguardar la salud de los miembros de su familia prefieren ir menos veces a supermercados, farmacias y otros lugares para adquirir sus compras.

Para evitar contagios algunas empezaron a comprar por medio de canales digitales debido a que prefieren pagar un valor adicional y que alguien más haga sus compras para prevenir y cuidarse del virus.

Todas las entrevistadas manifestaron que no creen que sus gastos hayan disminuido que creen que estos se mantienen o aumentaron debido a que migraron ciertos gastos innecesarios a gastos necesarios.

Las entrevistadas creen que la prioridad es la alimentación, la salud y una correcta higiene tanto personal como de su hogar que puede ayudar a contrarrestar la carga del virus, el dinero utilizado en vanidades o en actividades de ocio en el caso de ciertas entrevistadas se migró a los productos priorizados por cada hogar.

La mayoría de las entrevistadas manifestaron que dejaron de comprar maquillaje o de adquirir servicios relacionados a los mismos por el hecho de que no se puede salir y que deben de cuidarse $\mathrm{y}$ destinar esos recursos a otros rubros.

Por el tema de la ansiedad muchas buscaron realizar ciertas cosas por sus medios como el teñirse el cabello, el aplicarse tratamientos caseros o de marcas sean estos capilares o faciales, pintarse las uñas, pintarse las cejas con la finalidad de tener un medio de distracción.

Las entrevistadas más jóvenes manifestaron que recurrieron a servicios de maquillaje permanente o semipermanente en sus cejas con la finalidad de sentirse algo maquilladas debido a que las cejas son el marco del rostro.

Las mujeres que se encuentran que salen o tienen reuniones de trabajo donde deben tener sus cámaras encendidas si utilizan maquillaje, pero de 
manera leve debido a que creen que la imagen es importante en el ámbito laboral, sobre todo.

El ticket de compra y uso de maquillaje disminuyó considerablemente debido a que no salen de sus hogares por ende no necesitan maquillarse mientas que sólo una manifestó que su promedio de compra y el uso de maquillajes se redujo a la mitad debido a que por su actividad laboral si se maquilla de manera frecuente.

Las panelistas mencionaron que no compran productos de maquillaje o belleza por marcas, sino que se basan en recomendaciones sean estas de expertos, influencers o de conocidos para la adquisición de ciertos productos. Al comprar maquillaje el precio no es un factor tan relevante sino la calidad, el precio es lo de menos no todos los productos económicos son de mala calidad, también se destacó que pueden comprar ciertos productos por promociones y en el caso de obtener buenos resultados después volver adquirir los mismos incluso a su precio normal.

Creen que terminada la pandemia puede ser que vuelvan a su rutina de maquillaje completa, ahora solo se enfocan en resaltar la parte visible que es su mirada, pero se destacó que en la industria de belleza se está volviendo más práctica.

Indicaron que el maquillaje permanente para rostro, labios, cejas y ojos será una tendencia altamente demandada a futuro debido al ahorro que se tiene en temas de tiempos y de productos por adquirir estos servicios que tienen duración de seis hasta dos años dependiendo del área y el tipo de piel de cada persona.

En el estrato económico alto se entrevistaron a cuatro mujeres que se encuentran trabajando y dos mujeres que ahora son amas de casa con edades comprendidas de 24 a 44 años, están manifestaron lo siguiente:

Las familias de economías medias altas y altas no se vieron afectadas en sus ingresos a excepción de una de las participantes que comunicó que su esposo había sido despedido por la pandemia, el resto continúa trabajando en su mayoría desde casa o en el caso de las que son amas de casa sus esposos han mantenido sus trabajos.

A pesar de que sus ingresos no se vieron tan afectados todas mencionaron que redujeron ciertos gastos sea en productos o servicios con la finalidad de ahorrar o de comprar más comida y medicina para el hogar, sobre todo las que son mamás debido a que los niños por ansiedad comen más veces.

Todas manifestaron que la pandemia fue un período de gran incertidumbre que los afecto a nivel emocional de manera fuerte por la pérdida de familiares o conocidos, en la actualidad no han dejado de cuidarse y mencionaron que aun estando vacunadas lo seguirán haciendo debido a que la salud e integridad de su familia es primordial.

La frecuencia de compra cambió dejaron de comprar de forma semanal o quincenal para comprar de forma mensual prefieren tener un stock de diversos productos por si existe un nuevo confinamiento o simplemente para evitar contagio.

Algunas de las participantes mencionaron que la pandemia las obligó a buscar nuevos mecanismos de compras un ejemplo de este es el uso de aplicaciones de delivery enfocadas en hacer sus compras en los supermercados y entregar la misma en sus hogares con el fin de exponerse menos, prefieren pagar un adicional que salir de sus hogares.

La pandemia las obligó a realizar compras estrategias a buscar lo prioritario y dejar ciertos lujos a un lado como la adquisición de productos relacionados a maquillaje, perfumes, joyas, todo ese dinero lo migraron a gastos de comida sea en la compra de productos básicos o de comida elaborada.

Las mujeres de este grupo manifestaron que su relación con el maquillaje no cambio mucho principalmente para las que trabajan debido a que de manera frecuente tienen reuniones laborales con cámaras prendidas lo que las obliga a estar maquilladas por tema de cuidar su imagen y apariencia, en esta interacción salió la conocida 
frase antes muerta que sencilla evidenciando que sus rutinas no han sido modificadas.

El consumo de productos relacionados a belleza o cuidado personal no se redujo, sino que hubo cambios de sus categorías si antes compraban maquillaje con mayor frecuencia ahora compran con mayor frecuencia productos para el cuidado de su piel sea esta en su cuerpo o rostro, las cremas son altamente demandadas por este estrato.

Algunas de las panelistas mencionaron que la ansiedad y el tratar de tener algo productivo que hacer en la pandemia experimentaron realizar ciertos procedimientos de belleza por sus propios medios como las uñas, aprender a maquillarse entre otros.

Es fundamental mencionar que la mitad de las mujeres de este grupo han recurrido al maquillaje permanente en la pandemia por tema de practicidad y ahorro de tiempo, además de que les permite verse maquilladas de forma continua por eso se han realizado técnicas de sombreado permanente en sus cejas, colocación de pestañas pelo a pelo con la intención de que su mirada que es lo único visible se vea diferente.

Una de las panelistas mencionó que se vio obligada a cambiar de marca en cuánto a maquillaje debido a que por las restricciones comerciales fue difícil para algunas empresas de esta industria continuar importando ciertos productos.

Los atributos para comprar maquillaje son calidad, funcionabilidad, duración, marca y en algunos casos precios debido a que asignan un presupuesto determinado a este rubro, pero todas utilizan maquillajes de gama media a alta, alguna de las marcas nombradas fueron L'Oreal, Palladio, Maybelline entre otras.

Existe una mayor preferencia en marcas en productos relacionados a bases en polvo $\mathrm{o}$ liquidas y en cuanto a correctores, en cuanto a rímel, sombras, labiales y otros productos no se guían por una marca en específico.
Las panelistas mencionan que compran los productos de maquillaje por recomendaciones de expertos o famosos, que les gusta revisar los reviews de internet para conocer todos los aspectos buenos y malos de un producto, esto es un factor importante para tomar la decisión de adquirir los mismos.

Todas llegaron a la conclusión de que una vez que todo sea como antes, aunque creen que para eso falta mucho tiempo aún, sus rutinas de maquillaje y su frecuencia de compra y uso de las mismas regresarán debido a que tendrán vida social nuevamente, pero muchas resaltan que continuarán probando nuevas técnicas de maquillaje permanente por ahorro de tiempos y de dinero debido a que se deja de adquirir ciertos productos.

Tabla 1

Resumen de hallazgos encontrados por grupo focal enfocado en NSE

\begin{tabular}{|c|c|c|c|}
\hline Principales Hallazgos & $\begin{array}{l}\text { NSE } \\
\text { Bajo }\end{array}$ & $\begin{array}{c}\text { NSE } \\
\text { Medio }\end{array}$ & $\begin{array}{l}\text { NSE } \\
\text { Alto }\end{array}$ \\
\hline $\begin{array}{l}\text { Aumento del gasto redistribuyendo los } \\
\text { mismo. }\end{array}$ & $\mathrm{Si}$ & $\mathrm{Si}$ & $\mathrm{Si}$ \\
\hline Priorización de los gastos & $\mathrm{Si}$ & $\mathrm{Si}$ & $\mathrm{Si}$ \\
\hline Conciencia hacia el ahorro & $\mathrm{Si}$ & $\mathrm{Si}$ & $\mathrm{Si}$ \\
\hline $\begin{array}{l}\text { Mayor consumo de productos de salud } \\
\text { y alimentación }\end{array}$ & $\mathrm{Si}$ & $\mathrm{Si}$ & $\mathrm{Si}$ \\
\hline Realización de compras inteligentes & $\mathrm{Si}$ & $\mathrm{Si}$ & $\mathrm{Si}$ \\
\hline $\begin{array}{l}\text { Impulso por comprar promociones o } \\
\text { productos con } \\
\text { (Incentivando al ahorro) }\end{array}$ & $\mathrm{Si}$ & $\mathrm{Si}$ & $\mathrm{Si}$ \\
\hline $\begin{array}{l}\text { Dejaron de usar maquillaje por la } \\
\text { pandemia }\end{array}$ & $\mathrm{Si}$ & $\mathrm{Si}$ & $\mathrm{Si}$ \\
\hline $\begin{array}{l}\text { Luego de la pandemia el uso de } \\
\text { maquillaje será igual que antes }\end{array}$ & No & No & No \\
\hline
\end{tabular}

Los hallazgos de todos los grupos son iguales en lo que se diferencian son en el presupuesto que destinan para sus compras, la frecuencia de compra, que marcas usan, en que canales compran y cuál es el mecanismo o la razón de compra es decir mediante que estrategia de marketing compran publicidad, recomendaciones, entre otros. 


\section{CONCLUSIONES}

Por medio de los grupos focales y la información proporcionada por Kantar se comprueba que la industria de belleza se vio afectada esto se corrobora con la opinión de María Fernanda León, presidenta ejecutiva de la Asociación ecuatoriana de empresas de productos cosméticos, higiene y absorbentes (Procosméticos), que informó que en Ecuador las fragancias, maquillaje y protectores solares tuvieron una reducción en ventas de un $35 \%$ y que los productos que menos impacto tuvieron dentro del sector de maquillajes fueron los productos para ojos también mencionó que la demanda de productos de higiene personal, incrementaron sus ventas un $60 \%$ en el caso de los geles antibacteriales que se consideran cosméticos desinfectantes estos incrementaron su consumo un $70 \%$. (El Comercio, 2020)

La información obtenida se complementa con artículos sobre esta industria donde se evidencia un comportamiento similar en otras partes del mundo, en América Latina, según un reporte de Fashionable Asia, plataforma de moda y belleza, "más de la mitad de las mujeres encuestadas (58\%) confiesa que invierte menos en belleza durante el confinamiento. Un $28 \%$ de ellas confiesa que mantiene la misma inversión que en la época sin confinamiento, y solo un $14 \%$ invierte más durante el confinamiento". De la misma forma, se desprende del estudio que no sólo la inversión de los shoppers ha disminuido, sino que además sus prioridades y preocupaciones obligarán a las empresas a comprender sus nuevos hábitos (InStoreView, 2021).

Lo expuesto por todos los grupos focales se complementa con que los consumidores se enfocan en la higiene y salud de su piel, dejándola descansar de productos pesados, con químicos y que tapen los poros, como el maquillaje. También buscan regresar a cuidados básicos pero efectivos, como un signo de "menos es más" (InsiderLatam, 2021).

Por medio de este artículo se evidencia que el hábito de consumo de los consumidores puede cambiar en poco tiempo y que es importante para las empresas de la industria de maquillaje y belleza que sepan escuchar las nuevas necesidades de sus clientes potenciales con la finalidad de mantenerse a la vanguardia de lo que estos requieren.

La innovación es un factor importante y que genera una ventaja competitiva para las empresas que lo realizan por lo cual es importante que se destinen recursos para realizar investigación y desarrollo en las empresas de la industria del cuidado personal.

\section{Limitaciones}

La principal limitación fue conseguir personas que estuvieran dispuestas a formas parte del grupo social por lo cual se tuvo que recurrir a incentivos para que las panelistas estén predispuestas a contestar las preguntas a realizar sin problema y sin mentir.

$\mathrm{Al}$ efectuar un grupo focal de manera digital es un poco más complejo debido a que no existe una fluidez muy amplia y no se puede hacer una valoración por medio de ciertos gestos corporales o fáciles si un miembro del panel estaba en el mismo por compromiso y no está realizando aportaciones de valor.

\section{Futuras líneas de investigación}

Es fundamental realizar grupos focales enfocados a más productos de la línea de cuidado personal para confirmar que los hallazgos encontrados no tienen un sesgo y la muestra si es representativa para el comportamiento a nivel país.

También se podría realizar grupo focales a hombres para conocer qué ha pasado con el comportamiento de compra de estos relacionados a los productos de la canasta de cuidado personal enfocados principalmente al área de maquillaje, perfumes y cremas. 


\section{REFERENCIAS}

BASF. (2020). La Nueva Normalidad: ¿Cómo COVID-19 cambiará la industria de Cuidado del Hogar y Limpieza Industrial e Institucional? Recuperado el Junio de 2021, de https://www.basf.com/mx/es/media/Noticias/PM X_2020/la-nueva-normalidad---como-covid-19cambiara-la-industria-de-cui.html

Bellash. (2015). Que es la Venta por Catalogo? Recuperado el Junio de 2021, de https://www.bellash.com/blogs/articulos/417075 85-que-es-la-venta-por-catalogo

Camargo, j. (2020). Cómo calcular la frecuencia de compra y 3 consejos para mejorarla. Obtenido de https://juandcamargo.com/como-calcular-lafrecuencia-de-compra-y-3-consejos-paramejorarla/

Campaña, B. (2021). ¿Qué son los Social Ads? Obtenido de https://www.iebschool.com/blog/que-son-socialads-publicidad-online/

Cardona, L. (2019). 35 tipos de estrategias de marketing que funcionan. Obtenido de https://www.cyberclick.es/numerical-blog/35tipos-de-estrategias-de-marketing-que-funcionan

Centro Europeo de Postgrado. (2021). ¿Qué es la medición de la satisfacción del cliente? Recuperado el Junio de 2021, de https://www.ceupe.com/blog/que-es-lamedicion-de-la-satisfaccion-del-cliente.html

Cera, C. (2021). ¿Cuáles son los diferentes canales de venta y cómo elegir los correctos? Obtenido https://www.appvizer.es/revista/relacioncliente/gestion-ventas/canales-de-venta

EAE Business School. (2021). Las 5 etapas del proceso de decisión del comprador. Recuperado el Junio de 2021, de https://www.eaeprogramas.es/blog/negocio/empr esa/las-5-etapas-del-proceso-de-decision-delcomprador
El Comercio. (2020). Tendencias cosméticas cambian por la pandemia. Obtenido de https://www.elcomercio.com/tendencias/cambioconsumo-cosmeticos-pandemia-covid19.html

El Universo. (2020). Industria alimentaria creció durante la pandemia. Recuperado el Junio de 2021, de https://www.eluniverso.com/noticias/2020/07/25 /nota/7917966/industria-alimentaria-creciodurante-pandemia/

Emprendedores.es. (2021). Técnicas de marketing para provocar la compra por impulso. Recuperado el Junio de 2021, de https://www.emprendedores.es/gestion/provocarcompra-por-impulso-tecnicas-marketing/

Espinosa, R. (2019). Piramide de Maslow ¿Qué es? niveles y ejemplos. Obtenido de https://robertoespinosa.es/2019/06/09/piramidede-maslow

Fondo Monetario Internacional. (2020). Ayudar al Ecuador a enfrentar la pandemia. Recuperado el Junio de 2021, de https://www.imf.org/es/News/Articles/2020/10/0 5/na100520-helping-ecuador-confront-thepandemic

García, I. (2018). Penetración en el Mercado, el KPI que mira cuantos clientes nos compran. Obtenido de https://www.garciareal.com/2018/09/04/penetrac ion-en-el-mercado-el-kpi-que-mira-cuantosclientes-nos-compran/

Giraldo, V. (2019). Marketing de contenidos: la estrategia digital que debes conocer a profundidad. Obtenido de https://rockcontent.com/es/blog/marketing-decontenidos/

Hamui-Sutton, A., \& Varela-Ruiz, M. (2013). La técnica de grupos focales. Obtenido de https://www.elsevier.es/es-revista-investigacioneducacion-medica-343-articulo-la-tecnicagrupos-focales-S2007505713726838

Higuerey, E. (2019). Comercio electrónico: conoce todo sobre este modelo de negocios y 
cuáles son sus ventajas. Obtenido de https://rockcontent.com/es/blog/comercioelectronico/

Hootsuite. (2019). Estrategias de marketing en las redes sociales: 8 tácticas que no te puedes perder. Recuperado el Junio de 2021, de https://blog.hootsuite.com/es/estrategias-demarketing-en-redes-sociales/

Hotmart. (2021). ¿Cuáles son los canales de venta más eficientes hoy en día? Recuperado el Junio de 2021, de https://blog.hotmart.com/es/canales-deventa/

Hotmart. (2021). Marketplace: descubre las posibilidades que ofrece este negocio. Recuperado el Junio de 2021, de https://blog.hotmart.com/es/que-es-marketplace/

InboundCycle. (2021). ¿Qué es el marketing de influencers y cómo puede ayudarte en la promoción de tu marca? Recuperado el Junio de 2021, https://www.inboundcycle.com/diccionariomarketing-online/marketing-influencers

InsiderLatam. (2021). Rutinas de belleza: los cambios que trajo la pandemia. Obtenido de https://insiderlatam.com/rutinas-de-belleza-loscambios-que-trajo-la-pandemia/

Instituto Nacional de Estadísticas y Censos. (2011). Principales resultados. Recuperado el Junio de 2021, de https://www.ecuadorencifras.gob.ec//documento s/web-

inec/Estadisticas_Sociales/Encuesta_Estratificaci on_Nivel_Socioeconomico/111220_NSE_Presen tacion.pdf

InStoreView. (2021). Nueva belleza: tendencias clave de la industria post pandemia. Obtenido de https://www.instoreview.com/blog/nuevabelleza-tendencias-clave-de-la-industria-postpandemia

Lisboa, R. (2019). Focus Group: ¿qué es esta metodología y cómo aplicarla a tu estrategia de marketing? Obtenido de https://rockcontent.com/es/blog/focus-group/
Nardi, G. (2017). Estrategias de promoción al consumidor. Obtenido de https://desnudandoelmarketing.com/estrategiasde-promocion/

Nonato, L. (2019). Ticket promedio: qué es y cómo calcular. Obtenido de https://rockcontent.com/es/blog/ticket-promedio/

Organizacion Mundial de la Salud. (2020). Cronología de la respuesta de la OMS a la COVID-19. Recuperado el Junio de 2021, de https://www.who.int/es/news/item/29-06-2020covidtimeline

Ortiz, J. (2020). Investigación exploratoria: tipos, metodología y ejemplos. Obtenido de https://www.lifeder.com/investigacionexploratoria/

Peiró, R. (2019). Página web. Obtenido de es un documento accesible desde cualquier navegador con acceso a internet, y que puede incluir audio, vídeo, texto y sus diferentes combinaciones.

Peiró, R. (2020). Supermercado. Obtenido de https://economipedia.com/definiciones/supermer cado.html

Peña, O. (2020). Analítica de datos. Obtenido de https://www.poli.edu.co/blog/poliverso/analiticade-datos

Peralta, E. (2021). ¿Qué es el Social Media Marketing? Obtenido de https://www.genwords.com/blog/social-mediamarketing\#\%C2\%BFQue_es_Social_Media_Ma rketing_o_Marketing_en_Redes_Sociales

Pérez, J., \& Merino, M. (2021). Gráfico . Obtenido de https://definicion.de/grafico/

Pursell, S. (2020). Publicidad de boca en boca: qué es, por qué funciona y cómo impulsarla. Obtenido de https://blog.hubspot.es/marketing/publicidadboca-en-boca

QuestionPro. (2021). ¿Cuáles son los tipos de comportamiento de compra? Recuperado el Junio de

2021 , 
https://www.questionpro.com/blog/es/tipos-decomportamiento-de-compra/

QuestionPro. (2021). Cómo descubrir los hábitos de consumo de tus clientes. Recuperado el Junio de 2021, de https://www.questionpro.com/blog/es/habitosde-consumo/

QuestionPro. (2021). Panel de investigación de mercado. Qué es y cómo utilizarlo. Recuperado el Junio de 2021, de https://www.questionpro.com/blog/es/panel-deinvestigacion-de-mercado/

Ramírez, A. (2018). ¿Cuáles son las diferencias entre promoción, descuento, oferta y liquidación? Obtenido de https://www.informabtl.com/cualeslas-diferencias-promocion-descuento-ofertaliquidacion/

Rockcontent. (2019). Redes sociales: qué son, cómo funcionan, qué tipos existen y cómo influyen en las estrategias de Marketing. Recuperado el Junio de 2021, de https://rockcontent.com/es/blog/que-son-lasredes-sociales/

Rodríguez, A. (2019). PROCESO DE DECISIÓN DE COMPRA: QUÉ ES Y ETAPAS. Obtenido de https://somechat.es/proceso-de-decision-decompra-que-es-y-etapas/

Sima, A. (2020). CANAL MODERNO VS CANAL TRADICIONAL: DIFERENCIAS. Obtenido de https://clubdeltrade.com/blog/canalmoderno-canal-tradicional/

Solomon, M. (2013). Comportamiento del consumidor. Naucalpan de Juárez: Pearson Educación de México.

Sordo, A. (2020). Customer Journey: las 5 etapas del proceso de decisión de compra. Obtenido de https://blog.hubspot.es/marketing/etapas-dedecision-de-compra-del-consumidor

Sordo, A. (2020). Los 10 factores que afectan el comportamiento de tus consumidores. Obtenido de https://blog.hubspot.es/marketing/factorescomportamiento-del-consumidor
Sordo, A. (2021). Estrategias de publicidad: definición, tipos y ejemplos. Obtenido de https://blog.hubspot.es/marketing/estrategias-depublicidad

Stanpa. (2021). El cuidado personal sufre un impacto del $10 \%$ como consecuencia de la pandemia en 2020. Recuperado el Junio de 2021, de https://www.stanpa.com/notas-prensa/elcuidado-personal-sufre-un-impacto-del-10como-consecuencia-de-la-pandemia-en-2020/

Suprapak. (2020). LOS RETOS DE LA INDUSTRIA COSMÉTICA DURANTE LA PANDEMIA. Recuperado el Junio de 2021, de https://suprapak.com/retos-industria-cosmeticapandemia/

Tetrapack. (2020). Los consumidores recurren a productos que aumentan la inmunidad durante una pandemia. Recuperado el Junio de 2021, de https://www.tetrapak.com/es-ec/insights/casesarticles/consumers-immunity-boosting-productspandemic

Viñarás, E. (2021). KPI en marketing: qué es y métricas más importantes. Obtenido de https://www.cyberclick.es/numerical-blog/quees-un-kpi-en-tu-estrategia-de-marketing 